\title{
Engineered mesenchymal cell-based patches as controlled VEGF delivery systems to induce
}

extrinsic angiogenesis

Stefano Boccardo ${ }^{1,2^{*}}$; Emanuele Gaudiello ${ }^{1 *}$; Ludovic Melly ${ }^{1}$; Giulia Cerino ${ }^{1}$; Davide Ricci ${ }^{3}$; Ivan Martin $^{1}$; Friedrich Eckstein ${ }^{1}$; Andrea Banfi ${ }^{1 *}$, Anna Marsano $^{1 *}$

${ }^{1}$ Department of Surgery, University Hospital Basel, Basel, Switzerland; Department of

Biomedicine, University of Basel, Basel, Switzerland

${ }^{2}$ Musculoskeletal Disease Area, Novartis Institutes for BioMedical Research, Basel, Switzerland

${ }^{3}$ CTNSC, Istituto Italiano di Tecnologia, Ferrara, Italy

*Equally contributing authors

Corresponding author: Anna Marsano, $\mathrm{PhD}$

Cardiac Surgery and Engineering Group, Departments of Biomedicine and Surgery, University of Basel, University Hospital Basel, Hebelstrasse 20, 4031.

Phone: +41(0)613287448

Email: Anna.Marsano@usb.ch 


\begin{abstract}
Therapeutic over-expression of Vascular Endothelial Growth Factor (VEGF) by transduced progenitors is a promising strategy to efficiently induce angiogenesis in ischemic tissues (e.g. limb muscle and myocardium) but tight control over the micro-environmental distribution of dose is required to avoid induction of angioma-like tumors. Therapeutic VEGF release was achieved by purified transduced adipose mesenchymal stromal cells (ASC) that homogeneously produce specific VEGF levels, inducing only normal angiogenesis after injection in non-ischemic tissues. However, the therapeutic potential of this approach mostly in the cardiac field is limited by the poor cell survival and the restricted area of effect confined to the cell-injection site. The implantation of cells previously organized in vitro in 3D engineered tissues could overcome these issues. Here we hypothesized that collagen sponge-based construct (patch), generated by ASC expressing controlled VEGF levels, can function as delivery device to induce angiogenesis in surrounding areas (extrinsic vascularization). A 7-mm-thick acellular collagen scaffold (empty), sutured beneath the patch, provided a controlled and reproducible model to clearly investigate the ongoing angiogenesis in subcutaneous mice pockets. VEGF-expressing ASC significantly increased the capillary in-growth inside both the patch itself and the empty scaffold compared to naïve cells, leading to significantly improved survival of implanted cells.
\end{abstract}

These data suggest that this strategy confers control (i) on angiogenesis efficacy and safety by means of ASC expressing therapeutic VEGF levels and (ii) over the treated area through the specific localization in an engineered collagen sponge-based patch.

Keywords: angiogenesis; tissue engineering; VEGF; genetically modified cells. 


\section{Introduction}

Promising pro-angiogenic strategies based on the delivery of potent angiogenic factors, as the vascular endothelial growth factor-A (VEGF), have been investigated to induce growth of blood vessels in ischemic tissues [1,2]. Rapid and efficient restoration of the microvasculature is particularly crucial and challenging after a myocardial infarction to rescue organ function [3]. Phase I and II clinical studies based on VEGF gene therapy showed inconsistent outcomes in cardiac functionality in patients after myocardial infarction, likely due to the low local transfection efficacy, resulting in limited amount and duration of the protein expression $[4,5]$. In fact, VEGF expression needs to be controlled within a defined therapeutic window [6] and sustained for a period of at least four weeks to allow the maturation and the stabilization of the newly formed blood vessels [7]. Control over the dose and timing of VEGF release could be achieved by functionalized biomaterials $[8,9,10]$ though with some shortcomings with regard to the limited factor supply and the unforeseeable potency of the local host immune reaction [11]. A cell-based gene therapy approach could overcome such limitations by inducing a suitable cell population to overexpress the specific angiogenic gene [12]. However, the microenvironmental dose of VEGF needs to be controlled in order to avoid the growth of aberrant vascular structures and angioma-like tumors, which would jeopardize clinical benefit $[13,14]$. In order to ensure controlled expression of homogeneous VEGF levels in vivo, we recently developed a high-throughput FACS-based technology to rapidly purify populations of transduced human adipose-derived mesenchymal stromal cells (ASC) that produce only specific desired VEGF levels $[15,16]$. However, another key issue that severely limits the therapeutic efficacy of this approach is the poor survival of delivered ASC after direct intra-myocardial injection [17, 18, 19]. The global treatment of all affected areas is also difficult by cell injections. Delivery of cells following in vitro organization into engineered constructs may provide superior control over the targeted 
cardiac segments and enhance the implanted cell survival, thereby sustaining the delivery of the therapeutic signals $[20,21]$. Therefore, in this study as proof of principle we aim to combine a purified population of genetically modified ASC and a scaffold-based engineered patch to provide both spatial and dose control in VEGF delivery. Although three major splicing isoforms of VEGF exist (comprised of 120/121, 164/165, and 188/189 amino acids in rodents and humans, respectively) [22], in this study we focused on $\mathrm{VEGF}_{164}$ because it is the only isoform able to induce a physiologically patterned vasculature as a single factor in the absence of the others [23].

During in vitro 3D culture, bioreactors for direct perfusion of the medium were used to promote the reproducibility and the quality of cell-based patches by uniform distribution during seeding and efficient provision of nutrients and oxygen in mm-thick porous collagen scaffolds [24]. In particular, we hypothesized that FACS-purified VEGF-expressing ASC precultured in perfusion bioreactors on $3 \mathrm{D}$ sponges can provide a controlled and sustained VEGF release to induce safe and efficient angiogenesis both within the engineered patch (intrinsic) and in a surrounding area of clinically relevant size (extrinsic). Since the extrinsic angiogenic potential of the engineered tissues was assessed in a subcutaneous mouse model, we used a several mm-thick cell-free collagen scaffold (empty) to provide a standardized and controlled region in which to investigate dynamics of vascular growth.

\section{Materials and methods}

\subsection{Cell transduction and sorting}

Adipose tissue was obtained from a healthy donor undergoing plastic surgery after informed consent and according to a protocol approved by the Ethical Committee of Basel University Hospital. All investigations conform to the Declaration of Helsinki. Tissue was minced and digested with $0.15 \%$ collagenase (Worthington Biochemical Corporation, Lakewood, NJ) in PBS at $37{ }^{\circ} \mathrm{C}$ under continuous shaking for 60 minutes. Released cells were strained through a 
$70 \mu \mathrm{m}$ nylon mesh, plated at a density of $10^{5}$ cells $/ \mathrm{cm}^{2}$ and cultured in high-glucose DMEM medium with $10 \%$ FBS. After 4 days, cells were transduced with retroviral vectors expressing rat $\mathrm{VEGF}_{164}$, linked in a bicistronic expression cassette to a truncated version of rat CD8a as a convenient FACS-sortable surface marker, as previously described [15]. After 12 days, transduced ASC at p0 were FACS-sorted with a BD Influx Cell Sorter (BD Biosciences, Basel, Switzerland) with a gate determined by a reference clonal population of murine skeletal myoblasts expressing safe and specific VEGF levels [16]. Two different cell groups were generated: (i) naïve ASC as control and (ii) cells expressing a specific safe VEGF level (VEGF-ASC).

\subsection{Patch preparation and in vivo implantation}

Engineered constructs (patches) were generated using either (1) naïve ASC or (2) VEGFASC. Bovine type I collagen scaffold (Ultrafoam ${ }^{\mathrm{TM}}$, Bard; size: $12 \mathrm{~mm}$ diameter $3 \mathrm{~mm}$ thickness) were placed in the bioreactor chamber as previously described [25]. Briefly, ASC $\left(2.2 \times 10^{6}\right.$ cells/construct) were seeded and cultured throughout the inner $8 \mathrm{~mm}$ of the scaffold by a perfusion-based system at a speed of $3 \mathrm{ml} / \mathrm{min}$ for the first 18 hours and at $0.15 \mathrm{ml} / \mathrm{min}$ for the subsequent 5 days. Patches were then removed from the bioreactor, cut with $8 \mathrm{~mm}$ punch to remove the not perfused rim of collagen and stitched with 2 points of surgical suture to a $8 \mathrm{~mm}$ diameter and $7 \mathrm{~mm}$ thick empty scaffold (empty scaffold).

Animals were treated in compliance with Swiss Federal guidelines for animal welfare and all procedures were approved by the Veterinary Office of the Canton Bern (Bern, Switzerland) and conform to the Directive 2010/63/EU of the European Parliament. Male nude mice (Hsd: RH-rnu/rnu) were anesthetized by inhalation using a mixture of oxygen $(0.6 \mathrm{~L} / \mathrm{min})$ and Isoflurane (1.5-3 vol\%). Grafts were implanted in subcutaneous pockets in the back of the mouse. After 7, 14 and 28 days post-implantation, mice were anesthetized by intraperitoneal injection of a mixture of Ketamine $(100 \mathrm{mg} / \mathrm{Kg})$ and Xylazin $(10 \mathrm{mg} / \mathrm{Kg})$ prior sacrifice by total body vascular perfusion of $1 \%$ paraformaldehyde (PFA). The harvested constructs were 
further fixed in PFA 4\% for 5 hours and left in 30\% sucrose in PBS overnight, before embedding in OCT compound (Sakura Finetek, Torrance, CA) and freezing in liquid nitrogen-cooled isopentane vapors.

\subsection{DNA assay}

For DNA assay, patches $(n=3)$ were collected from bioreactors, washed twice in PBS and digested overnight at $56{ }^{\circ} \mathrm{C}$ with protease $\mathrm{K}(0.5 \mathrm{~mL}$ of $1 \mathrm{mg} / \mathrm{mL}$ protease $\mathrm{K}$ in $50 \mathrm{mM}$ Tris with $1 \mathrm{mM}$ EDTA, $1 \mathrm{mM}$ iodoacetamide, and $10 \mathrm{mg} / \mathrm{mL}$ pepstatin-A). Total DNA amount was measured with the CyQUANT ${ }^{\circledR}$ Cell Proliferation Assay Kit (Life Technologies). DNA concentration was determined by reading the absorbance value at $485 \mathrm{~nm}$ using a Sinergy $\mathrm{H} 1$ Hybrid Reader (Biotek instruments Gmbh, Lucern, Switzerland).

\subsection{Flow cytometer analysis}

For phenotypic characterization of 3D-cultured VEGF-ASC, patches ( $n=3$ per condition) were digested in $0.15 \%$ collagenase (Worthington Biochemical Corporation, Lakewood, NJ), liberated cells were collected by centrifugation and incubated for $20 \mathrm{~min}$ on ice, in PBS with 5\% bovine serum albumin (BSA). The antibodies used were: CD90-FITC, CD73-PE, CD31FITC, CD45-FITC, IgG1-FITC, IgG1-PE, IgG-APC (all from Becton and Dickinson Company, Franklin Lakes, NJ), CD105-FITC (Serotec Ltd. Oxford, UK), and VEGF-R2FITC (R\&D Systems). All the antibodies were used at a dilution of 1:50, except CD105FITC, which was used at 1:20. Data were acquired with a FACSCalibur flow cytometer (BD Biosciences). For cell cycle analysis cells, retrieved from the patch, were fixed in $70 \%$ ethanol and incubated in a solution containing propidium iodide (Sigma-Aldrich) $10 \mu \mathrm{g} / \mathrm{ml}$ in PBS and RNAase $10 \mu \mathrm{g} / \mathrm{ml}$ for 20 minutes at $37^{\circ} \mathrm{C}$. Data were acquired with Fortessa II cytometer (BD Biosciences) and analysed by using FlowJo software (Tree Star, Ashland, OR, USA).

\subsection{MTT staining}

Qualitative spatial distribution of living cells was assessed by MTT (3(4,5-dimethylthiazol-2-yl)2,5-diphenyltetrazolium bromide; Sigma-Aldrich) staining. Active mitochondria convert this 
tetrazolium salt into an insoluble formazan which stains cells purple. Samples were cut in half, rinsed in PBS and incubated at $37{ }^{\circ} \mathrm{C}$ for 2 hours with $3 \mathrm{ml}$ of $0.12 \mathrm{mM}$ MTT.

\subsection{Histology}

All histological analyses were performed on frozen sections. For basic histomorphological evaluation, sections were stained with hematoxylin and eosin (H\&E) according to standard protocols. Color images were acquired with an Olympus BX63 microscope (Olympus, Volketswil, Switzerland) or a Zeiss LSM710 confocal microscope (Zeiss, Oberkochen, Germany). For immunofluorescence analyses cryosections were incubated for 1 hour in $0.3 \%$ Triton X-100 and 2\% normal goat serum in PBS, and then for 1 hour in the following primary antibodies and dilutions: (1) anti-CD31 (AbDSerotec, Düsseldorf, Germany) at 1:100, (2) antiNG2 (Millipore, Zug, Switzerland) at 1:200, (3) anti- $\alpha$ Smooth-Muscle Actin (Sigma-Aldrich, Basel, Switzerland) at 1:400, (4) anti-cleaved Caspase-3 (Asp175) (Cell Signaling Technology, Allschwil, Switzerland) at 1:100, (5) anti-Ki-67 (AbCam, Cambridge, UK) at 1:200, (6) antiHuman Nuclei clone 235-1 (Millipore, Billerica, MA, USA) at 1:100, anti-laminin (AbCam, Cambridge, UK) at 1:400, anti-VE-Cadherin (Santa Cruz Biotechnology Dallas, Texas, USA) at 1:200. All secondary antibodies were used at 1:200 for 1 hour incubation (Life Technologies, Basel, Switzerland). Nuclei were stained using DAPI (Invitrogen, Switzerland). All antibodies were diluted in $0.3 \%$ Triton $\mathrm{X}-100$ and $2 \%$ normal goat serum in PBS.

\subsection{Vessel length density quantification}

The amount of blood vessels was quantified on images taken from CD31-stained sections by determining the total vessel length density (VLD). Six representative fluorescent images per sample (4 samples per group) were acquired with a 20x objective on an Olympus BX61 microscope (Olympus, Münster, Germany), both in the patch and in the empty scaffold areas (at the center and at the border). The total length of the capillaries was measured by tracing the CD31-positive vessels using CellSens software (Soft Imaging System, Münster, Germany). The 
vessel length density was then determined by dividing the total vessel length by the area of each field.

\subsection{Human cell quantification}

Human cell survival was evaluated by assessing the human cell density at 28 days post implantation. Six representative fluorescent images per sample ( 2 samples per group; $n=12$ ) were acquired both in the patch and in the area of the empty scaffold below the patch $(100 \mu \mathrm{m}$ away from the border with the patch) with 20x magnification on an Olympus BX63 microscope (Olympus, Volketswil, Switzerland) on cross-sections. Cells positive for human nuclei staining (see paragraph 2.6) were counted using ImageJ 1.47 software (Research Service Branch, NIH, USA) and divided by the image area to obtain the cell density. The in vivo percentage of proliferating ASC at 28 days was assessed by dividing the number of double positive Human Nuclei $(\mathrm{HuNu})$ and $\mathrm{Ki}-67$ cells by the total amount of human cells.

\subsection{Elisa assay}

The rate of VEGF production by ASC was quantified in the supernatant of $2 \mathrm{D}$ cell culture using a Quantikine rat VEGF immunoassay enzyme-linked immunosorbent assay (ELISA) kit (R\&D Systems), as previously described [16]. Briefly, 4 milliliters of fresh medium was added on Naïve or VEGF-ASCs cultured in 60-mm dishes in quadruplicate for $4 \mathrm{~h}$, collected, filtered, and frozen. Results were normalized by the number of cells in each dish and the time of incubation and expressed as $\mathrm{ng} / 10^{6}$ cells/day.

For the estimation of VEGF concentrations in the patches during 3D culture, patches were removed from the bioreactor after 4 days and immediately frozen. After homogenization of the patch in a PBS $+1 \%$ Triton X-100 buffer, VEGF concentration was assessed with an ELISA kit (R\&D Systems) and values were normalized by the estimated number of cells (according to the average of cell seeding efficiency) and the amount of the medium present in the bioreactor and expressed as $\mathrm{ng} / 10^{6}$ cells.

\subsection{Statistics}


Data were analyzed with the statistical software Prism 4.0a (GraphPad, La Jolla, CA) and SPSS 17.0 (IBM, Armonk, NY). Data are presented as means \pm standard deviation. Comparison between groups was performed using a one-way ANOVA with Bonferroni correction for the VEGF quantification and a mixed-effects model for the angiogenesis quantification. $\mathrm{P}<0.05$ was considered statistically significant.

\section{Results}

\subsection{Engineered tissue characterization}

Direct perfusion of culture medium through the scaffold allowed a uniform cell distribution through the entire thickness (top, center and bottom) of the collagen sponge (Fig 1A). Quantification of the nuclei by image analysis confirmed the homogeneous cell distribution (about 300-350 cells $/ \mathrm{mm}^{2}$ ) throughout the entire construct cross-section (Fig. 1B). Seeding efficiency was measured by quantifying the amount of genomic DNA of the total amount of cells used for the seeding (as reference) and that retrieved from the scaffold 1 day after the loading and after further 5 days of perfusion culture (Fig 1C). The seeding efficiency was very high $(98.3 \% \pm 0.5 \%$ after 1 day compared with the reference amount of DNA).

Macroscopic images of patches stained for metabolically active cells by using MTT excluded the presence of a necrotic central core (Fig. 1 D), further confirming the homogeneous cell viability in the scaffold after 5 days of culture.

\subsection{Effect of VEGF expression on ASC}

VEGF $_{164}$ is a potent mitogen and also affects cell survival, with effects being conserved across species. Therefore, we investigated its possible effects on human ASC proliferation, apoptosis and phenotype. No significant difference was found in the cell density after 5 days of culture in constructs generated by VEGF-expressing and naïve ASC (Fig. 2A). Furthermore, cell cycle analysis with propidium iodide by cytofluorimetry showed that the 
majority of the cells (about 80\%) was in G0/G1 phase in both experimental groups (Fig. 2BC), thus confirming that VEGF expression did not increase ASC proliferation. Low doubling rates for both naïve and VEGF-ASC were also confirmed by immunofluorescence staining for Ki-67 protein, a marker expressed in actively proliferating cells in all phases of the cell cycle (Fig. 2D). VEGF expression by ASC appeared also not to affect cell apoptosis, since no relevant differences were found in the expression of caspase 3 (CAS-3), a key-protein in the apoptotic process (Fig. 2E). Cytofluorimetry analyses for hematopoietic (CD45 $)$ mesenchymal $\left(\mathrm{CD} 73^{+}, \mathrm{CD} 90^{+}\right)$and endothelial $\left(\mathrm{CD} 31^{+}, \mathrm{VEGFR}-2^{+}\right)$markers showed, as expected, that the near-totality of the ASC were of mesenchymal/stromal origin, with no hematopoietic cells and a minor amount of endothelium. Importantly, no relevant changes in the ASC population composition occurred when VEGF was expressed (Fig. 2F). Monolayerexpanded transduced ASC produced $58.5 \pm 11 \mathrm{ng} / 10^{6}$ cells/day of rat VEGF (Fig. 2G), corresponding to the levels previously found to be safe and efficacious [16]. Patches generated with ASC were then sutured on the top of empty collagen scaffold of critical size in order to assess their in vivo intrinsic and extrinsic angiogenic potential (Fig. $2 \mathrm{H})$.

\subsection{In vivo vascularization of the patch}

Before implantation, the amount of VEGF produced by transduced ASC after 3D perfusion culture was measured and was comparable to the rate of production measured during monolayer culture $\left(41.9 \pm 7.1 \mathrm{ng} / 10^{6}\right.$ cells $)$. Upon implantation of mm-thick 3D patches, a prompt vascularization of the cell-seeded construct is essential to guarantee enough oxygen and nutrient provision to avoid cell loss. Moreover, in the scenario of angiogenesis induction through cell-based gene therapy, efficient survival of implanted cells is also crucial to achieve a sustained VEGF release. To investigate the kinetics of patch vascularization, we implanted cell-seeded patches ectopically in a subcutaneous pocket in nude mice and the resulting vascularization was analyzed at different time points (4, 14 and 28 days) by staining for the 
endothelial marker CD31. Vessel ingrowth was observed already after 4 days in VEGFexpressing patches and increased at 14 and 28 days in vivo (Fig. 3A-B). Branching points (white arrows in Fig. 3A), that indicate the formation of a well inter-connected and physiological microvascular network, were observed almost exclusively in VEGF-expressing patches and increased with time in vivo (Fig. 3A). Naïve ASC constructs contained few endothelial cells in the center and only few vessels were observed even at late time points (Fig 3A). The vessel length density (VLD) was statistically significantly greater at all time points in VEGF patches (Fig. 2B). Endothelial structure density in naïve cell-based constructs was similar at all time points. After 4 days in vivo, the vessel length density was 6-fold higher in the VEGF-ASC compared to control cell. A remarkable 4.9 fold increase in VLD was observed at 14 days and was maintained up to 28 days in the VEGF-ASC patches compared to the naive. In VEGF-releasing patches, the newly formed blood vessels were also tightly surrounded by pericytes, as shown by immunofluorescence staining for NG2, confirming the formation of mature capillaries (Fig. 3C-D). Further, only morphologically normal capillary networks were observed and no instances of enlarged, aberrant angioma-like vascular structures could be detected. Taken together, these results indicate that patches seeded with transduced and purified ASC expressing controlled VEGF levels support the intrinsic induction of safe, efficient and mature angiogenesis.

\subsection{Angiogenesis induction outside the patch}

To investigate the extrinsic pro-angiogenic potential of the engineered patches, the induction of vessel ingrowth was investigated in an empty collagen scaffold sutured underneath the cellseeded patch and with a critical thickness of $7 \mathrm{~mm}$. Host blood vessels reached the center of the empty scaffold only when VEGF-expressing cells were seeded in the patch sutured on top (Fig. 4A). In fact, at 28 days almost no vessels were present both in the center and at the border of the empty collagen scaffolds underneath the naïve ASC-based patches (Fig. 4A). At 4 days no host blood vessels reached yet the center of the acellular scaffold in both conditions 
(Fig 4A) and a negligible amount of sprouting capillaries was observed in the control sponge at later time points in vivo (Fig. 4A-B). On the contrary, VEGF-expressing patches induced a significant ingrowth of capillaries in the center of the underlying empty scaffold already by 14 days, which was maintained at 28 days (Fig. 4A-B, center). Host vessels start infiltrating the border of the empty patches of both conditions at 4 days. However, only in the presence of VEGF-releasing patches the VLD increased significantly at 14 and 28 days after implantation (Fig 4A-B, border). VEGF-expressing patches induced the formation of a mature capillary network with a physiologically branched morphology and the majority of new vessels were tightly associated with pericytes (Fig 4C). Moreover, no growth of aberrant structures was observed at either of the three time points, as expected with the delivery of controlled VEGF levels. Taken together, these results indicate that only VEGF-expressing patches have the ability to efficiently induce safe and mature angiogenesis in an avascular volume placed at a significant distance from the engineered patch itself.

\subsection{In vivo cell survival and migration.}

To assess the functional consequences of the observed vascular ingrowth, we evaluated the survival of the human ASC seeded on the patches. After 28 days in vivo, control patches contained a lower amount of engrafted human cells compared to VEGF-expressing patches (Fig. 5A). Quantification of human cells (identified as staining positive for the Human Nuclei ( $\mathrm{HuNu}$ ) antigen) cells showed that the density of human ASC in the presence of VEGF expression was significantly greater than in control conditions, with an 11-fold increase (Fig. 5B). Interestingly, the absolute cell density of VEGF-expressing ASC was similar to the one measured before implantation (Fig. 1B), showing that VEGF expression completely prevented the cell loss that took place in control conditions instead. Most of the implanted cells were retained inside the patch, with very low cell migration in the area of the empty scaffold just next to the patch (Fig. 5C-D). No human cells were detected in the distal portion of the empty scaffold as well as in the surrounding host tissue in any condition. After 28 days in vivo, most 
of the implanted cells did not differentiate into a vascular lineage, as human cells could not be observed inside the vessel basement membrane, identified by laminin, which defines the limit between vascular cells (endothelium and pericytes) and the surrounding tissue (Figure 5E). Blood vessels were therefore essentially of mouse origin. Lastly, only very few implanted ASC were proliferating after 28 days in vivo, with no difference between naïve or VEGFexpressing cells, as shown by the quantification of human cells positive for Ki-67 (Figure 5F).

\section{Discussion and conclusions}

In this study, we showed that a patch seeded with a purified population of transduced adipose tissue-derived progenitor cells could function not only to attract intrinsic vascularization, but also as a device for delivery of controlled VEGF levels to induce extrinsic angiogenesis. Several strategies have been investigated in the past years to promote angiogenesis for the treatment of ischemic diseases or the rapid vascularization of engineered tissue implants. The successful generation of in vitro engineered substitutes (e.g. skin, bone, fat and muscles) cannot be translated clinically if a prompt in vivo vascularization strategy is not envisioned to allow implanted cell survival, assuring their function also upon implantation [26]. Efficient vascularization of engineered tissues is particularly important in the case of critical-size (several mm-thick) constructs. The most commonly adopted strategy consists in prevascularizing the engineered tissues by co-culturing mature or progenitor endothelial cells with mesenchymal stem cells or perivascular cells [27] or by the use of isolated [28] or preformed vessels [29]. Moreover the use of several biomaterials functionalized to release proangiogenic factors is an alternative promising method that is under investigation [30,31]. Our approach, based on the long-term over-expression of VEGF from implanted cells, favors the recruitment of blood vessels from the host, guaranteeing already after 4 days upon implant a dense vascularization of the entire cell-seeded construct (Fig. 3). On the opposite, patches prepared with naive ASC showed a few endothelial structures after 4 days: the fact that these 
were predominantly seen in the center of the patch, rather than at the border with the host tissue, suggests that these early structures may depend on the few endothelial cells present in the ASC populations (Fig. 2F). Notably, the higher vascular density in VEGF patches compared to naïve ASC was maintained at 14 days, suggesting that VEGF expression was necessary for effective vascularization of the engineered patch. 28 days after implantation, vascular density was not further increased in VEGF-ASC patches and the newly induced capillary networks were associated with normal pericytes, indicating that they have achieved maturation and stabilization, as well as that angiogenesis induced by controlled VEGF levels is a self-limiting, non-progressive process. Particularly attractive is the perspective to implement this angiogenic strategy for the generation of different engineered tissue substitutes (e.g. muscle, bone) by co-culturing VEGF-expressing cells with a suitable progenitor cell source [32].

A consistent body of literature both in skeletal muscle and myocardium previously found that controlling the VEGF dose at the microenvironmental level, to express only therapeutic levels that induce efficacious and normal angiogenesis, is crucial to avoid the formation of aberrant structures, since VEGF remains tightly localized in the matrix around expressing cells $[13,14,17]$. By correlating the production of VEGF with a FACS-sortable marker it is possible to select and purify specific populations that homogeneously express only a desired safe and effective level of VEGF [16]. It is however possible that the specific microenvironment of the 3D patch, in terms of scaffold composition and newly deposited extracellular matrix, might influence the spatial distribution of produced VEGF. Therefore, future studies will be necessary to investigate both the possibility of avoiding FACS-purification and the therapeutic outcome of delivering non-purified transduced ASC expressing heterogeneous VEGF levels.

Beyond the ability to attract vascularization inside the patch, we hypothesized that a patch seeded with VEGF-expressing cells could also act as a factor-delivery device and induce 
angiogenesis in the surrounding tissue. To test this hypothesis, we sutured beneath the patch an empty collagen scaffold of critical size (7-mm thick and 8-mm diameter). At 4 days after implantation no vascular structures were observed in the inner part of the empty scaffold. Moreover the infiltration that was measured at the border was similar in the presence of both control and VEGF-expressing patches, suggesting no VEGF effect at this early time point. However, by 14 days a significant difference was observed in both regions of the empty scaffold: at the border vessel density increased 2- and 4-fold in the VEGF expressing conditions compared to naive (at 14 and 28 days, respectively), while at the center a striking 8- and 20-fold increase was observed in the VEGF-expressing conditions compared to naive (at 14 and 28 days, respectively). Remarkably, VEGF expression induced a similar vascular density both in the center and at the border by 14 days, whereas in its absence VLD in the center remained greatly lower than at the border at all time points. Furthermore, these extrinsic vascular networks reached maturation through association with pericytes, which is a necessary condition for their long-term persistence and functional efficacy. These observations indicate that: 1) VEGF diffused to the edge and through to the core of the empty scaffold, covering a quite unexpected distance from the source and guiding the recruitment of blood vessels from the host, and 2) sustained expression, ensured by the survival and the stable transduction of ASC with an integrating viral vector, was necessary to provide sufficient time for vessels to reach the core of the empty scaffold. In this experimental setup, ASC did not have a role in the formation of blood vessels, but they rather functioned as vehicle for VEGF delivery while embedded in the collagen scaffolds.

The engineered ASC-based patch described here represents an innovative device for controlled VEGF delivery that could be implemented to different fields, among which the treatment of cardiac ischemia might be a particularly suitable application, due to its specific requirements. In this future perspective, ASC are widely considered an ideal cell source for cardiac applications [18]. In fact, skeletal myoblasts have been previously investigated as an 
attractive cell source for cardiac regeneration thanks to their capability to form contractile myofibers. However, they cannot acquire a cardiomyocyte phenotype and, most importantly, they form electrically active fibers that do not couple with the surrounding myocardium, causing dangerous arrhythmic events [33]. On the other hand mesenchymal stromal cells, including ASC, do not form electrically active tissue and have been reported not to increase the risk of arrhythmias [34], providing therefore a more suitable cell source to deliver VEGF in the heart. In order to develop such an application, the functional properties of the extrinsic angiogenic potential described here would need to be further evaluated in an ischemic cardiac model to assess whether the sustained and controlled delivery of safe and effective VEGF levels to the ischemic tissue could improve perfusion, restoring local contractility and global cardiac function. Moreover, based on previous studies, the approach described here could also provide functional benefit through the further release of other paracrine factors, produced by ASCs, which can exert positive effects on cardiomyocyte survival and on the ventricle remodeling after myocardial infarction [20].

In conclusion, our findings provide a proof of principle for generating an efficient angiogenic patch that has the potential to be used clinically. However, before a possible clinical translation, alternative vectors should be considered, since retroviruses raise safety concerns of neoplastic transformation of transduced stem cells by insertional mutagenesis [35]. Selfinactivating lentiviral vectors with chromatin insulator elements represent a valid solution thanks to their capability to express a transgene in a stable and sustained manner, but without the oncogenic potential of retroviral vectors $[36,37]$.

\section{Acknowledgements}

The authors are grateful to Professor Michael Heberer for productive scientific discussions and financial support. This study was funded by the Swiss National Science Foundation grant 149274 to A.M. and grant 143898 to A.B. 


\section{References}

[1] P. Korpisalo, S. Ylä-Herttuala, Stimulation of functional vessel growth by gene therapy, Integr. Biol. (Camb.) 2 (2010) 102-12.

[2] B. H. Annex, Therapeutic angiogenesis for critical limb ischaemia, Nat. Rev. Cardiol. 10 (2013) 387-96.

[3] A.M. Van der Laan, J.J. Piek, N. van Royen, Targeting angiogenesis to restore the microcirculation after reperfused MI, Nat. Rev. Cardiol. 6 (2009) 515-23.

[4] M.C. Scimia, A.M. Gumpert, W.J. Koch, Cardiovascular gene therapy for myocardial infarction, Exp. Opin. Biol. Ther. 14 (2014) 183-95.

[5] S. Ylä-Herttuala, J.E. Markkanen, T.T. Rissanen, Gene therapy for ischemic cardiovascular diseases: some lessons learned from the first clinical trials, Trends Cardiovasc. Med. 14 (2004) 295-300.

[6] G. Von Degenfeld, A. Banfi, M.L. Springer, R.A. Wagner, J. Jacobi, C.R. Ozawa, M.J. Merchant, J.P. Cooke, H.M. Blau, Microenvironmental VEGF distribution is critical for stable and functional vessel growth in ischemia, FASEB J. 20 (2006) 2657-9.

[7] Y. Dor, V. Djonov, R. Abramovitch, A. Itin, G.I. Fishman, P. Carmeliet, G. Goelman, E. Keshet, Conditional switching of VEGF provides new insights into adult neovascularization and pro-angiogenic therapy. EMBO J. 21 (2002) 1939-47.

[8] Y. Miyagi, L.L. Chiu, M. Cimini, R.D. Weisel, M. Radisic, R.K. Li, Biodegradable collagen patch with covalently immobilized VEGF for myocardial repair, Biomaterials, 32 (2011) 1280-90.

[9] D. Odedra, L.L. Chiu, M. Shoichet, M. Radisic, Endothelial cells guided by immobilized gradients of vascular endothelial growth factor on porous collagen scaffolds, Acta Biomater. 7 (2011) 3027-35.

[10] V. Sacchi, R. Mittermayr, J. Hartinger, M.M. Martino, K.M. Lorentz, S. Wolbank, A.

Hofmann, R.A. Largo, J.S. Marschall, E. Groppa, R. Gianni-Barrera, M. Ehrbar, J.A. Hubbell, H. 
Redl, A. Banfi, Long-lasting fibrin matrices ensure stable and functional angiogenesis by highly tunable, sustained delivery of recombinant VEGF164, Proc Natl Acad. Sci. U S A 111 (2014) 69527.

[11] S.F. Badylak, T.W. Gilbert, Immune response to biologic scaffold materials, Semin. Immunol. 20 (2008) 109-16.

[12] H.H. Moon, M.K. Joo, H. Mok, M. Lee, K.C. Hwang, S.W. Kim, J.H. Jeong, D. Choi, S.H. Kim, MSC-based VEGF gene therapy in rat myocardial infarction model using facial amphipathic bile acid-conjugated polyethyleneimine, Biomaterials 35 (2014) 1744-54.

[13] C.R. Ozawa, A. Banfi, N.L. Glazer, G. Thurston, M.L. Springer, P.E. Kraft, D.M. McDonald, H.M. Blau, Microenvironmental VEGF concentration, not total dose, determines a threshold between normal and aberrant angiogenesis, J. Clin. Invest. 113 (2004) 516-27.

[14] H. Karvinen, E. Pasanen, T.T. Rissanen, P. Korpisalo, E. Vähäkangas, A. Jazwa, M. Giacca, S. Ylä-Herttuala, Long-term VEGF-A expression promotes aberrant angiogenesis and fibrosis in skeletal muscle, Gene Ther. 18 (2011) 1166-72.

[15] H. Misteli, T. Wolff, P. Füglistaler, R. Gianni-Barrera, L. Gürke, M. Heberer, A. Banfi, Highthroughput flow cytometry purification of transduced progenitors expressing defined levels of vascular endothelial growth factor induces controlled angiogenesis in vivo, Stem Cells 28 (2010). [16] U. Helmrich, A. Marsano, L. Melly, T. Wolff, L. Christ, M. Heberer, A. Scherberich, I. Martin, A. Banfi, Generation of human adult mesenchymal stromal/stem cells expressing defined xenogenic vascular endothelial growth factor levels by optimized transduction and flow cytometry purification, Tissue Eng. Part C Methods 18 (2012) 283-92.

[17] L.F. Melly, A. Marsano, A. Frobert, S. Boccardo, U. Helmrich, M. Heberer, F.S. Eckstein, T.P. Carrel, M.N. Giraud, H.T. Tevaearai, A. Banfi, Controlled angiogenesis in the heart by cellbased expression of specific vascular endothelial growth factor levels, Hum. Gene Ther. Methods 23 (2012) 346-56. 
[18] M. Mazo, V. Planat-Bénard, G. Abizanda, B. Pelacho, B. Léobon, J.J. Gavira, I. Peñuelas, A. Cemborain, L. Pénicaud, P. Laharrague, C. Joffre, M. Boisson, M. Ecay, M. Collantes, J. Barba, L. Casteilla, F. Prósper, Transplantation of adipose derived stromal cells is associated with functional improvement in a rat model of chronic myocardial infarction, Eur. J. Heart Fail. 10 (2008) 454-62. [19] H. Wei, T.H. Ooi, G. Tan, S.Y. Lim, L. Qian, P. Wong, W. Shim, Cell delivery and tracking in post-myocardial infarction cardiac stem cell therapy: an introduction for clinical researchers. Heart Fail. Rev. 15 (2010) 1-14.

[20] H. Hamdi, V. Planat-Benard, A. Bel, E. Puymirat, R. Geha, L. Pidial, H. Nematalla, V. Bellamy, P. Bouaziz, S. Peyrard, L. Casteilla, P. Bruneval, A.A. Hagège, O. Agbulut, P. Menasché, Epicardial adipose stem cell sheets results in greater post-infarction survival than intramyocardial injections, Cardiovasc. Res. 91 (2011) 483-91.

[21] H. Hamdi, A. Furuta, V. Bellamy, A. Bel, E. Puymirat, S. Peyrard, O. Agbulut, P. Menasché, Cell delivery: intramyocardial injections or epicardial deposition? A head-to-head comparison, Ann. Thorac. Surg. 87 (2009) 1196-203.

[22] J. E. Park, G. A. Keller N. Ferrara, The vascular endothelial growth factor (VEGF) isoforms: differential deposition into the subepithelial extracellular matrix and bioactivity of extracellular matrix-bound VEGF, Mol. Biol. Cell. 4 (1993) 1317-26.

[23] C. Ruhrberg, H. Gerhardt, M. Golding, R. Watson, S. Ioannidou, H. Fujisawa, C. Betsholtz, D.T. Shima, Spatially restricted patterning cues provided by heparin-binding VEGF-A control blood vessel branching morphogenesis, Genes Dev. 16 (2002) 2684-98.

[24] R. Maidhof, A. Marsano, E.J. Lee, G. Vunjak-Novakovic, Perfusion seeding of channeled elastomeric scaffolds with myocytes and endothelial cells for cardiac tissue engineering, Biotechnol. Prog. 26 (2010) 565-72.

(3): 611-9. 
[25] G. Cerino, E. Gaudiello, T. Grussenmeyer, L. Melly, D. Massai, A. Banfi, I. Martin, F.

Eckstein, M. Grapow, A. Marsano, Three dimensional multi-cellular muscle-like tissue engineering in perfusion-based bioreactors, Biotechnol. Bioeng. 113 (2016) 226-36.

[26] M.W. Laschke, M.D. Menger, Prevascularization in tissue engineering: Current concepts and future directions, Biotechnol. Adv. 34 (2016) 112-21.

[27] J. Guerrero, S. Catros, S.M. Derkaoui, C. Lalande, R. Siadous, R. Bareille, N. Thébaud, L. Bordenave, O. Chassande, C. Le Visage, D. Letourneur, J. Amédée, Cell interactions between human progenitor-derived endothelial cells and human mesenchymal stem cells in a threedimensional macroporous polysaccharide-based scaffold promote osteogenesis, Acta Biomater. 9 (2013) 8200-13.

[28] X. Sun, W. Altalhi, S.S. Nunes, Vascularization strategies of engineered tissues and their application in cardiac regeneration, Adv. Drug Deliv. Rev. 96 (2016) 183-94.

[29] B. Zhang, M. Montgomery, M.D. Chamberlain, S. Ogawa, A. Korolj, A. Pahnke, L.A. Wells, S. Massé, J. Kim, L. Reis, A. Momen, S.S. Nunes, A.R. Wheeler, K. Nanthakumar, G. Keller, M.V. Sefton, M. Radisic, Biodegradable scaffold with built-in vasculature for organ-on-a-chip engineering and direct surgical anastomosis, Nat. Mater. 15 (2016) 669-78.

[30] M. Lovett, K. Lee, A. Edwards, D.L. Kaplan, Vascularization strategies for tissue engineering, Tissue Eng. Part B Rev. 15 (2009) 353-70.

[31] J.J. Kim, L. Hou, N.F. Huang, Vascularization of three-dimensional engineered tissues for regenerative medicine applications, Acta Biomater. (2016), Epub ahead of print.

[32] A. Marsano, R. Maidhof, J. Luo, K. Fujikara, E.E. Konofagou, A. Banfi, G. VunjakNovakovic, The effect of controlled expression of VEGF by transduced myoblasts in a cardiac patch on vascularization in a mouse model of myocardial infarction, Biomaterials 34 (2013) 393401. 
[33] P. Menasche, Cardiac cell therapy: lessons from clinical trials, J. Mol. Cell. Cardiol. 50 (2011) 258-65.

[34] P.L. Sanchez, R. Sanz-Ruiz, M.E. Fernandez-Santos, F. Fernandez-Viles, Cultured and freshly isolated adipose tissue-derived cells: fat years for cardiac stem cell therapy, Eur. Heart J 31 (2010) $394-7$.

[34] T.S. Yeh, Y.H. Fang, C.H. Lu, S.C. Chiu, C.L. Yeh, T.C. Yen, Y. Parfyonova, Y.C. Hu, Baculovirus-transduced, VEGF-expressing adipose-derived stem cell sheet for the treatment of myocardium infaretion, Biomaterials 35 (2014) 174-84.

[35] C. Baum, J. Dullmann, Z. Li, B. Fehse, J. Meyer, D.A. Williams, C. von Kalle, Side effects of retroviral gene transfer into hematopoietic stem cells, Blood 101 (2003) 2099-114.

[36] E. Montini, D. Cesana, M. Schmidt, F. Sanvito, C.C. Bartholomae, M. Ranzani, F. Benedicenti, L.S. Sergi, A.Ambrosi, M.Ponzoni, C.Doglioni, C.Di Serio, C.von Kalle, L. Naldini, The genotoxic potential of retroviral vectors is strongly modulated by vector design and integration site selection in a mouse model of HSC gene therapy, J. Clin. Invest. 119 (2009) 964-75.

[37] S.S. De Ravin, X. Wu, S. Moir, S. Anaya-O'Brien, N. Kwatemaa, P. Littel, N. Theobald, U. Choi, L. Su, M. Marquesen, D. Hilligoss, J. Lee, C.M. Buckner, K.A. Zarember, G. O'Connor, D. McVicar, D. Kuhns, R.E. Throm, S. Zhou, L.D. Notarangelo, I.C. Hanson, M.J. Cowan, E. Kang, C. Hadigan, M. Meagher, J.T. Gray, B.P. Sorrentino, H.L. Malech, Lentiviral hematopoietic stem cell gene therapy for X-linked severe combined immunodeficiency, Sci. Transl. Med. 8 (2016) 33557.

\section{Figure Legends}

Fig. 1 Characterization of the ASC-based patches. A) Representative pictures of hematoxylin and eosin staining taken at top, middle and bottom part of the scaffold (from the left to the right, respectively) (size bar $=100 \mu \mathrm{m})$. B) Quantification of cell density distribution by image analysis of 
9 random fields per sample ( $\mathrm{n}=3$ samples/condition). C) DNA amount Quantification of genomic DNA in the amount of cells used for the seeding (ref. after seeding) and present in constructs after 1 and 5 days of culture (n=3). D) Representative pictures of MTT staining on the entire scaffold after 5 days of culture (top and cross-section view at low and high magnification: from the left to the right; scale bar $=1 \mathrm{~mm})$. Graphs are showed as mean \pm Standard Deviation (SD).

Fig. 2 VEGF effects on ASC proliferation and phenotype in 3D in vitro culture. A) Quantification of cell density in VEGF and Naïve patches after 5 days of dynamic culture (image analysis performed on 6 random fields per sample; $n=3$ samples/condition). B) Representative histogram for flow cytometric analysis of cell cycle with propidium iodide emission for naïve (left) and VEGF- (right) ASC cultured on 3D collagen scaffold for 5 days. C) Quantification of percentage of cells present in either $\mathrm{G} 0 / \mathrm{G} 1$ or $\mathrm{S}$ or $\mathrm{G} 2 / \mathrm{M}$ cell cycle phase based on the flow cytometer analysis ( $\mathrm{n}=3$ per condition). D,E) Immunofluorescence staining for ki-67 and Cleaved Caspase-3 (green) and cell nuclei (blue) in the patches after 5 days in perfusion bioreactors (size bar $=20 \mu \mathrm{m}$ and $100 \mu \mathrm{m}$ respectively). F) Phenotypic characterization of VEGF and naïve ASC by flow cytometry analysis for hematopoietic (CD45), mesenchymal (CD90, CD73) and endothelial (CD31, VEGFR-2) markers. G) Quantification of the amount of rat VEGF released by transduced cells either in monolayer or in 3D perfusion-based culture. Data are presented as mean $\pm \mathrm{SD}(\mathrm{n}=4)$. H). Macroscopic view of the composed construct before implantation: the dashed black line distinguishes between the cell-based patch (patch) and the underneath cell-free collagen sponge (empty scaffold). At the border in blue there are the two surgical sutures (size bar $=5 \mathrm{~mm}$ ).

Fig. 3 In vivo vascularization of the ASC-based patches. A) Representative images of blood vessel density in patches seeded with naïve and VEGF-ASC after 4 and 28 days in vivo, assessed by immunofluorescence for CD31 (red) (size bar=20 $\mu \mathrm{m}$ ). B) Quantification of vessel length density (VLD) in the Naïve and VEGF patches at 4, 14 and 28 days upon implantation (image analysis performed on 6 random fields per samples $\mathrm{n}=4$ samples/condition; ${ }^{* *} \mathrm{p}<0.01 ;{ }^{* * *} \mathrm{p}<0.001$. C, D) Representative images of newly formed blood vessels stained for maturation markers at 28 
days in VEGF-ASC patch al low (C) and high (D) magnification. Immunofluorescence staining for cell nuclei (DAPI; Blu), endothelial cells (CD31; red), pericyte (NG2; green) and smooth muscle cells (SMA, cyan); (size bars $=100 \mu \mathrm{m}(\mathrm{C})$ and $20 \mu \mathrm{m}(\mathrm{D}))$.

Fig. 4 Induction of angiogenesis in the underneath empty collagen scaffold. A) Quantification of vessel length density (VLD) in the center and border area of the empty scaffold sutured beneath patches generated by either the Naïve or VEGF cells at 4, 14 and 28 days after implantation (image analysis on 6 random fields per area and per samples, $n=4$ samples/condition;

${ }^{* *} \mathrm{p}<0.01 ; * * * \mathrm{p}<0.001$. B) Representative images of blood vessel ingrowth in the center and border areas of the empty scaffolds 28 days after implantation, assessed by immunofluorescence for CD31 (red). Dashed line outlines the area of the empty scaffold which was in contact with the host tissue (size bar=20 $\mu \mathrm{m}$ ). C) Representative images of blood vessel maturation in the inner area of the empty scaffolds sutured beneath Naïve (left) or VEGF (right) patches 28 days after implantation, assessed by immunofluorescence staining for cell nuclei (DAPI; blu), endothelial cells (CD31; red), pericyte (NG2; green) and smooth muscle cells (SMA, cyan); (size bar= $20 \mu \mathrm{m})$.

Fig. 5 In vivo cell engraftment and migration. A) ASC survival at 28 days was assessed by immunofluorescence staining for human nuclei ( $\mathrm{HuNu}$, in red) while all nuclei were visualized by DAPI (blue). White arrows indicate human cell nuclei in the naïve (left) and VEGF (right) patches (size bar $20 \mu \mathrm{m}$ ). B) Quantification of human cell density in the naïve and VEGF patches at 28 days (image analysis on 6 random fields per sample; $n=z 4$ samples/condition; $* * * p<0.001) C$ ) (left): representative low-magnification image of specific human nuclei (red) and DAPI (blue) staining in the VEGF patch at 28 days. The white line represents the border between the patch and the empty scaffold (size bar $=200 \mu \mathrm{m}$ ). The small panels show higher magnification images of the areas in the

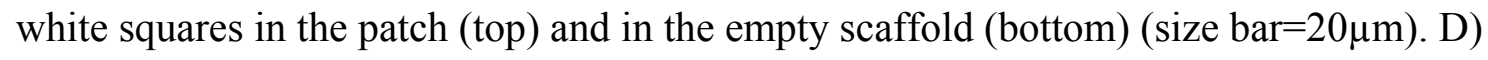

Quantification of human cell density at 28 days in the empty scaffold area below the naïve and VEGF patches (image analysis on 6 fields acquired randomly within $100 \mu \mathrm{m}$ from the border line; $\mathrm{n}=4$ samples/condition). E) Representative images of immunofluorescence staining for human cells 
(in red), VE-Cadherin (in green), laminin (in grey) and DAPI (in blue) of patches generated by Naive or VEGF-expressing ASC after 28 days in vivo (size bar=20 $\mu \mathrm{m}$ ). F) Graph representing the percentage of human cells that are proliferating after 28 days in vivo in patches generated by Naive or VEGF-expressing ASC (image analysis on 6 random fields per sample; $n=4$ samples/condition; no statistic difference). 


\section{VEGF-expressing Engineered ASC patches}
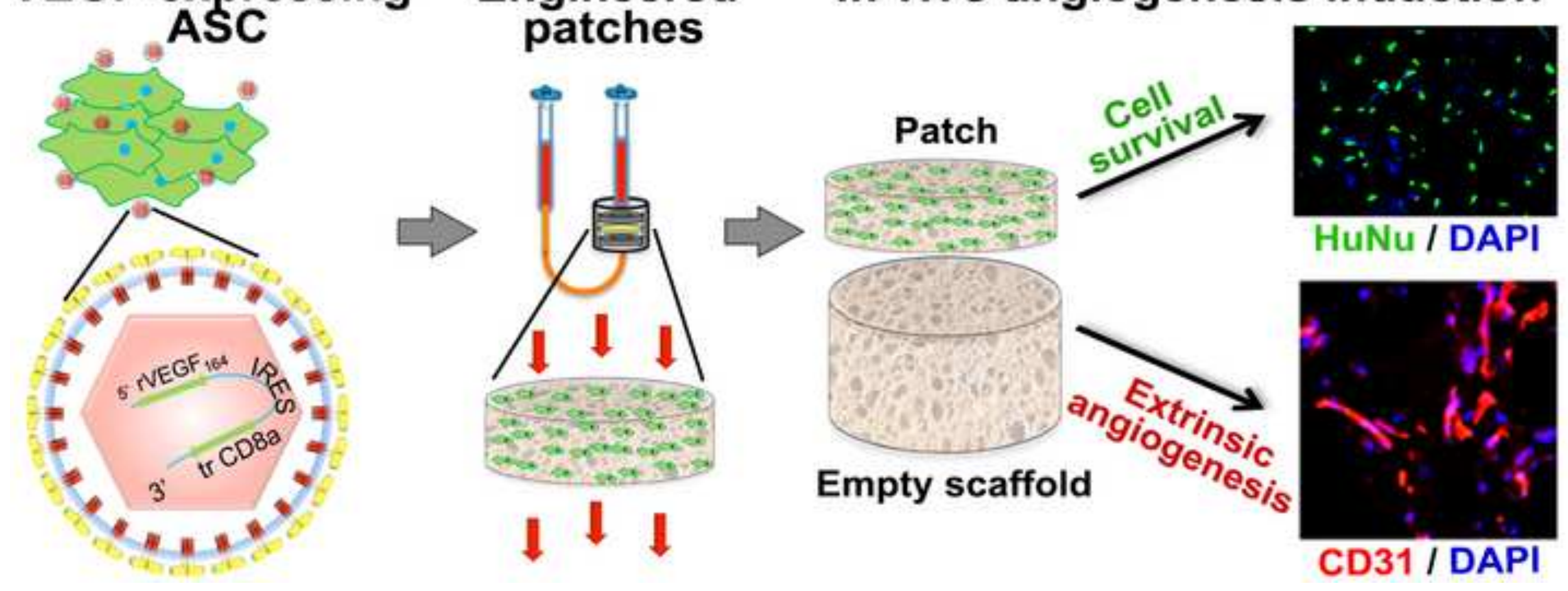

In vivo angiogenesis induction

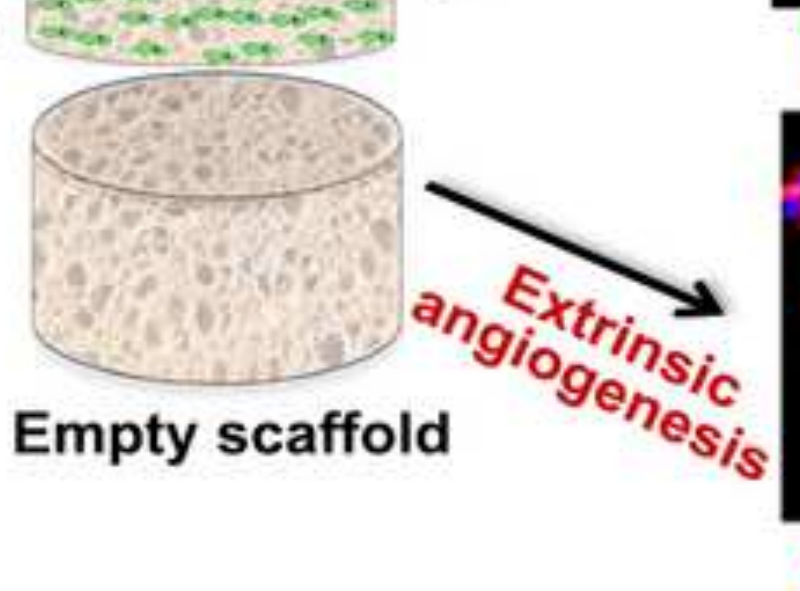

CD31 / DAPI 
Fig.1

A
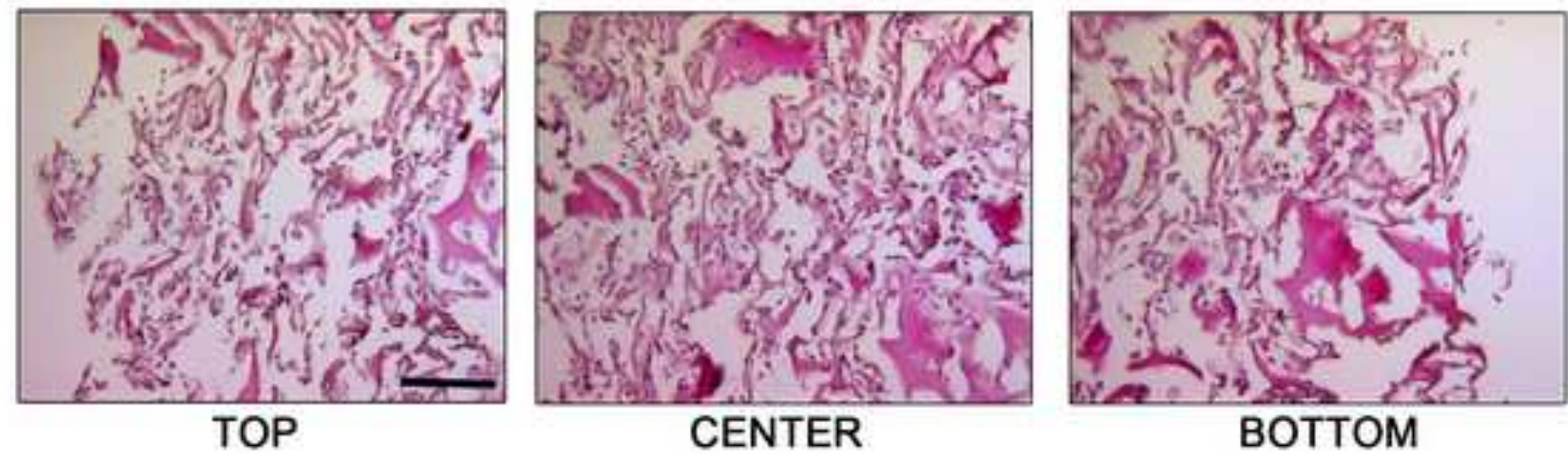

B

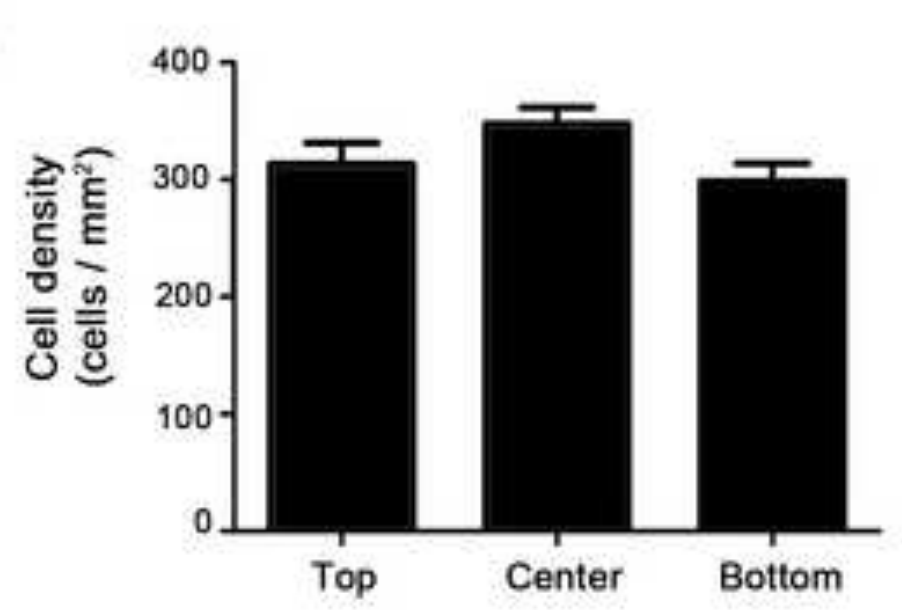

D

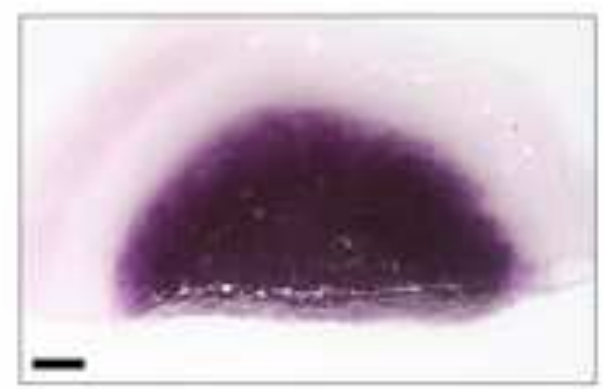

C

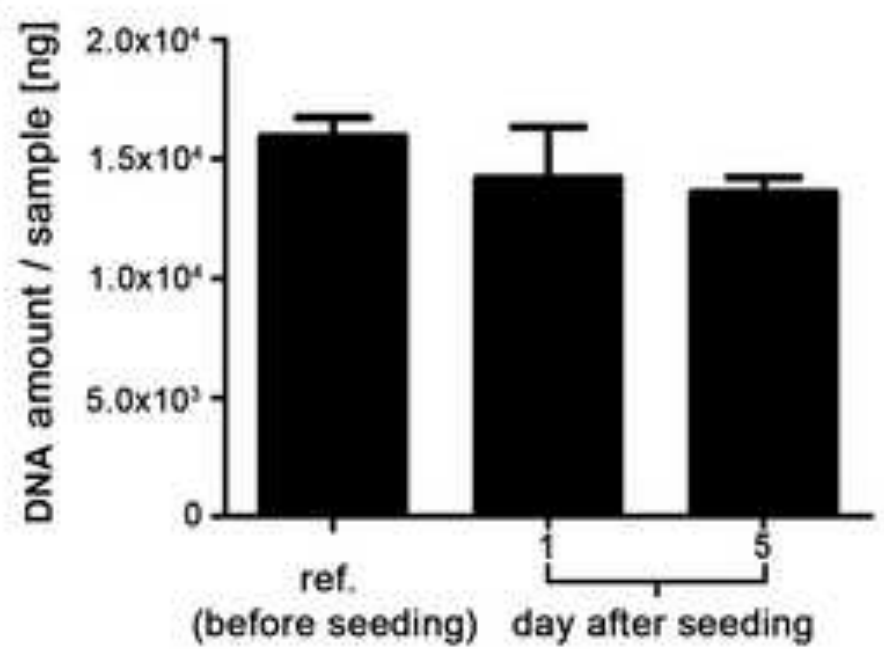

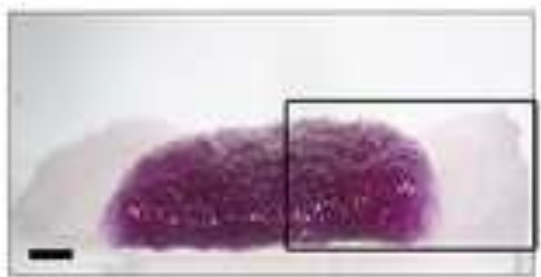

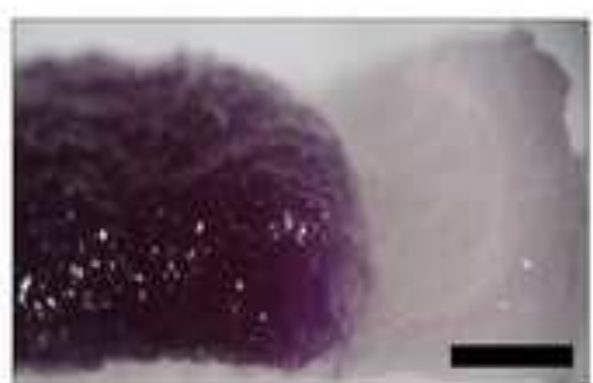



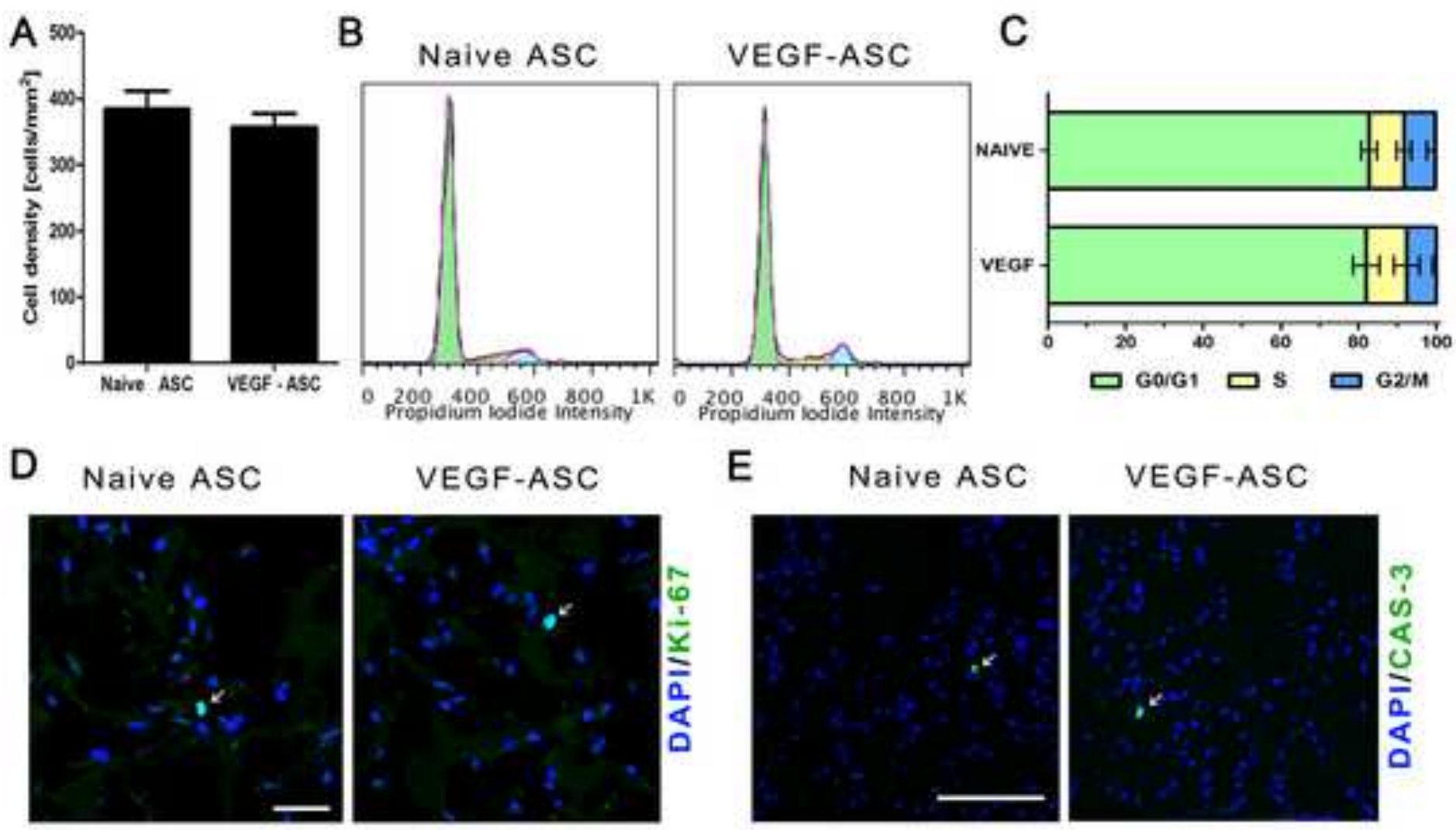

F
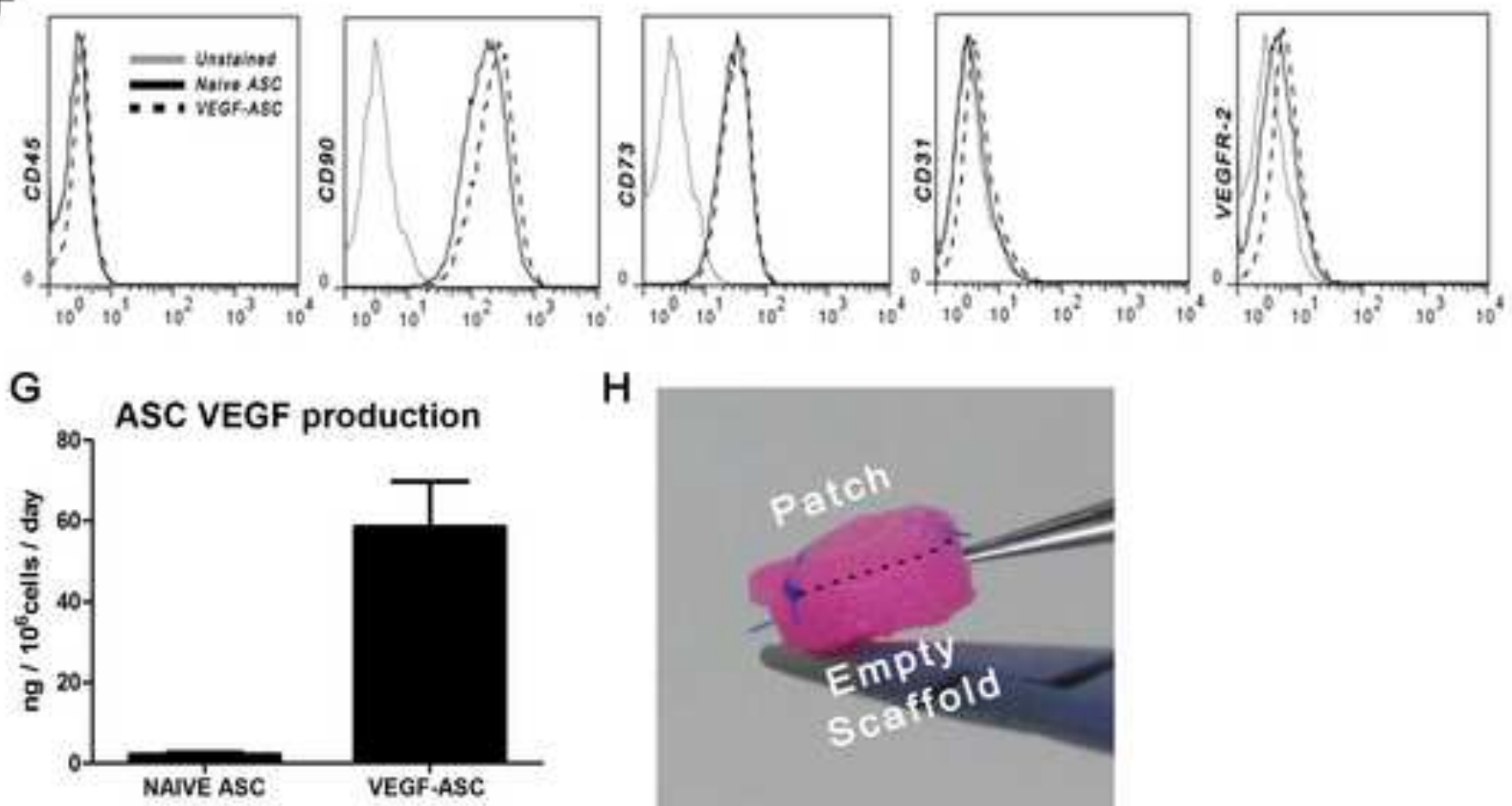

$\mathrm{H}$

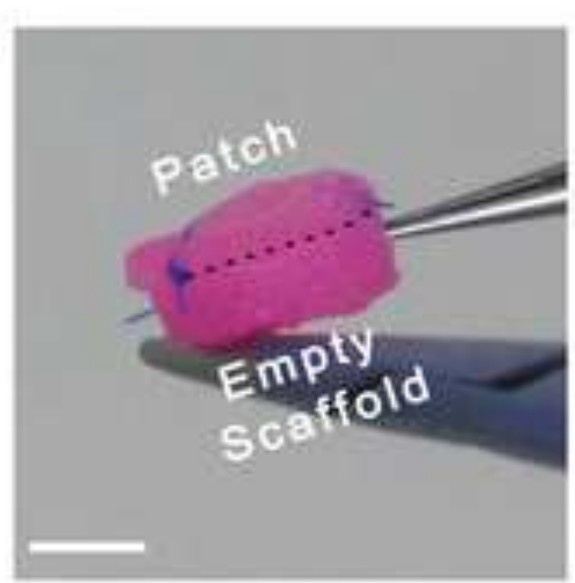




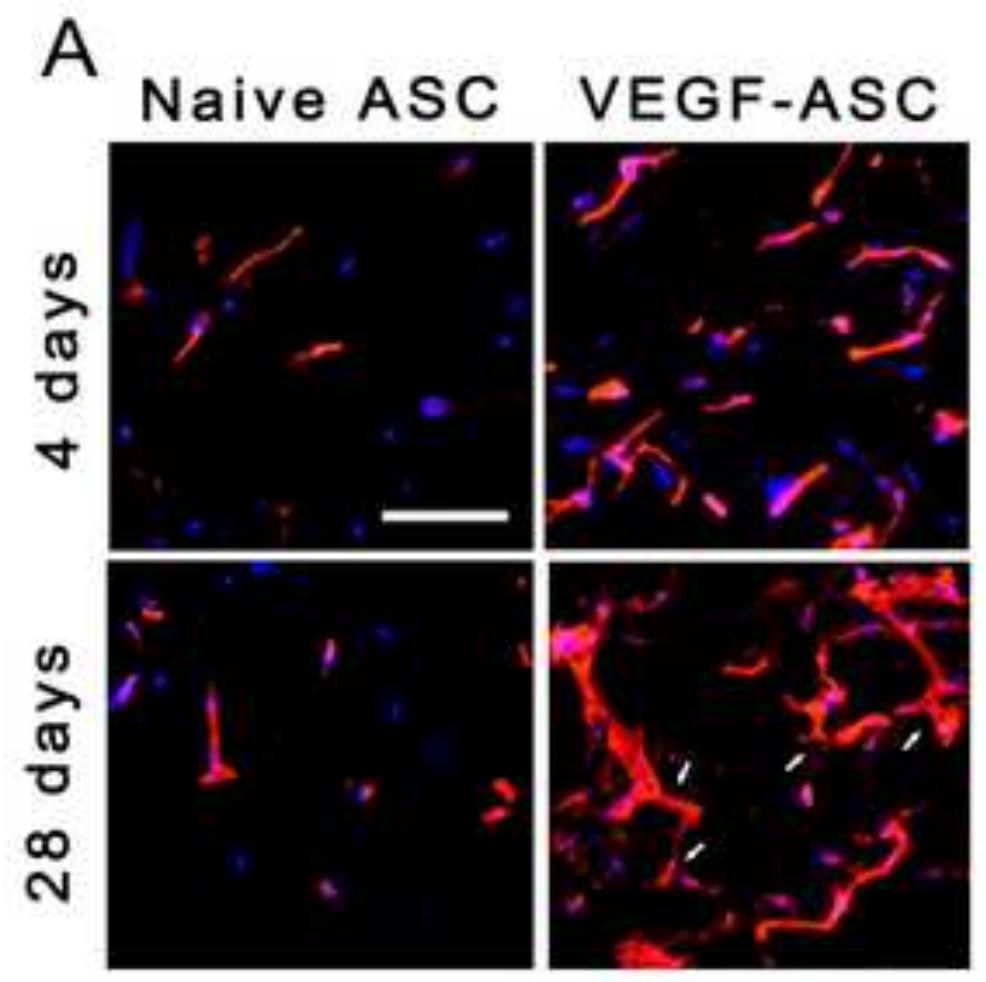

B

$\square$ Naive ASC

VEGF-ASC
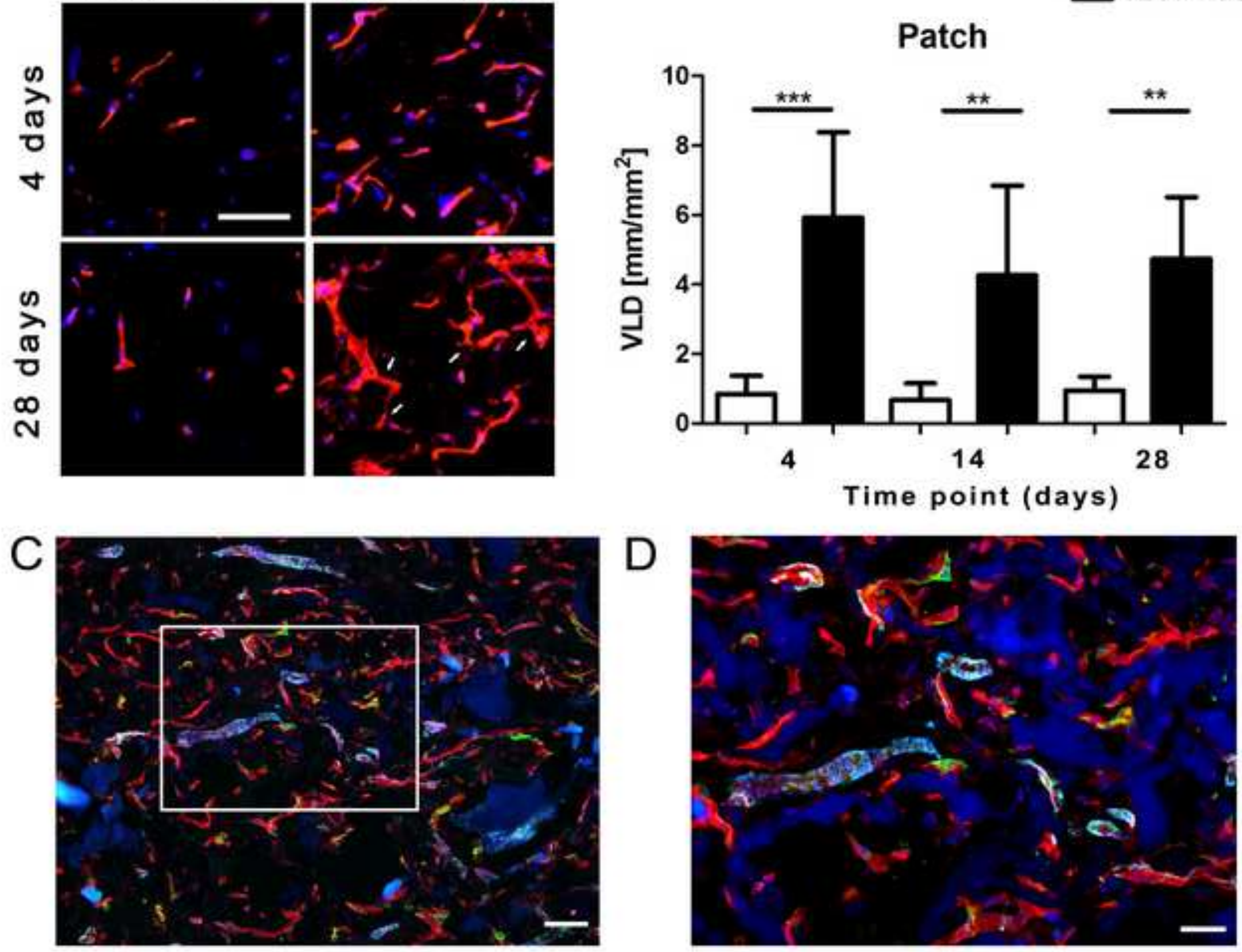
A
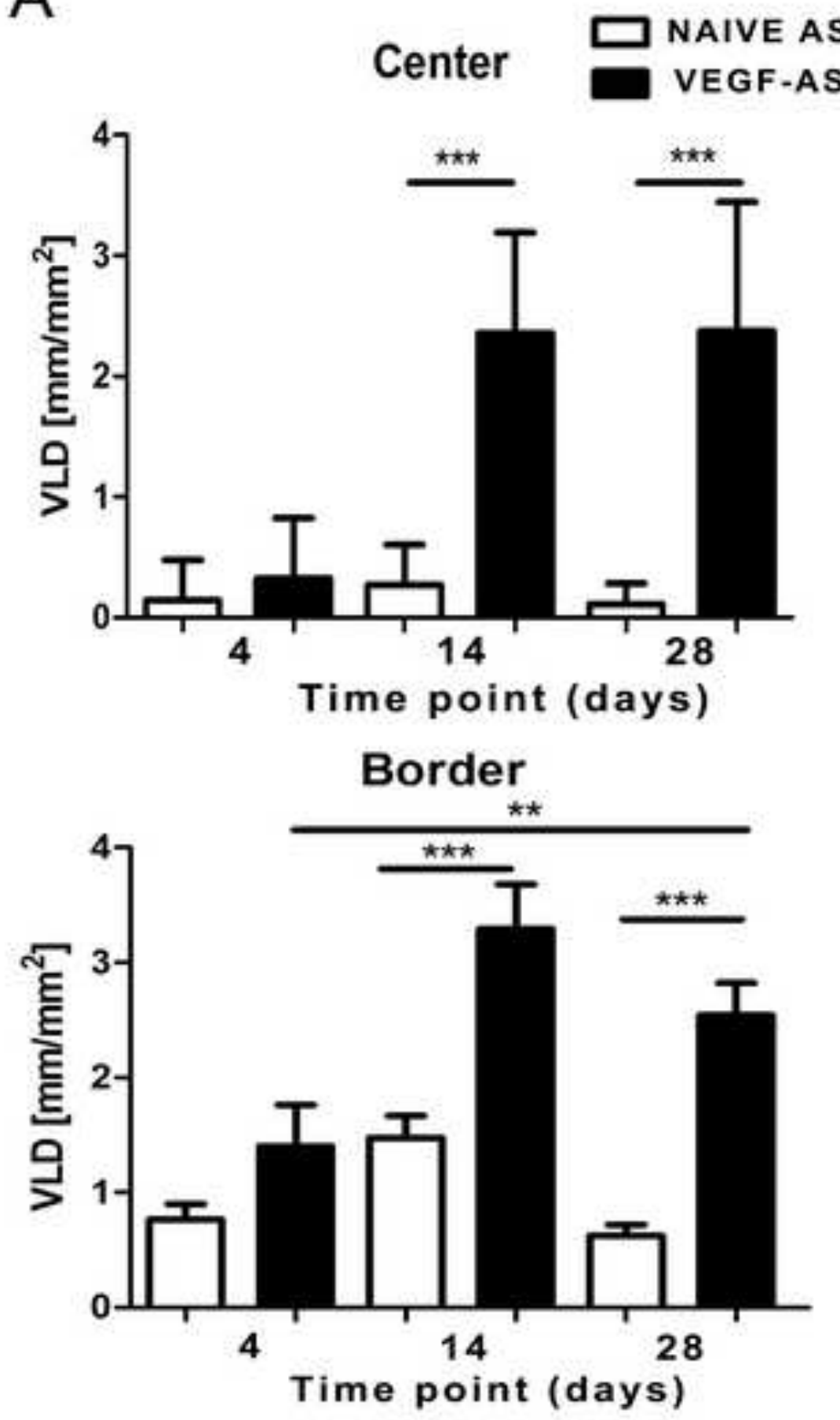

B Naive ASC

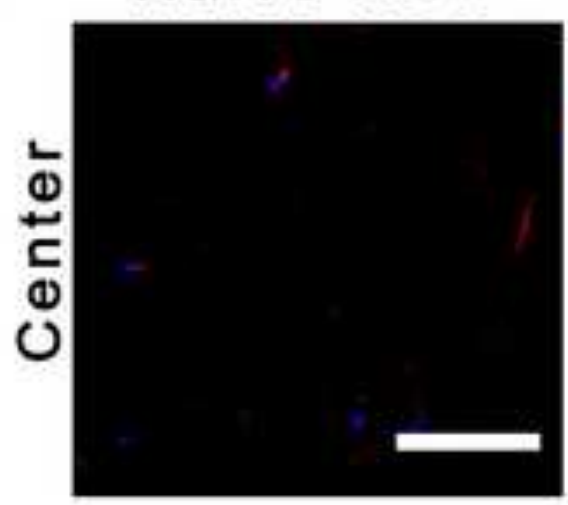

VEGF-ASC
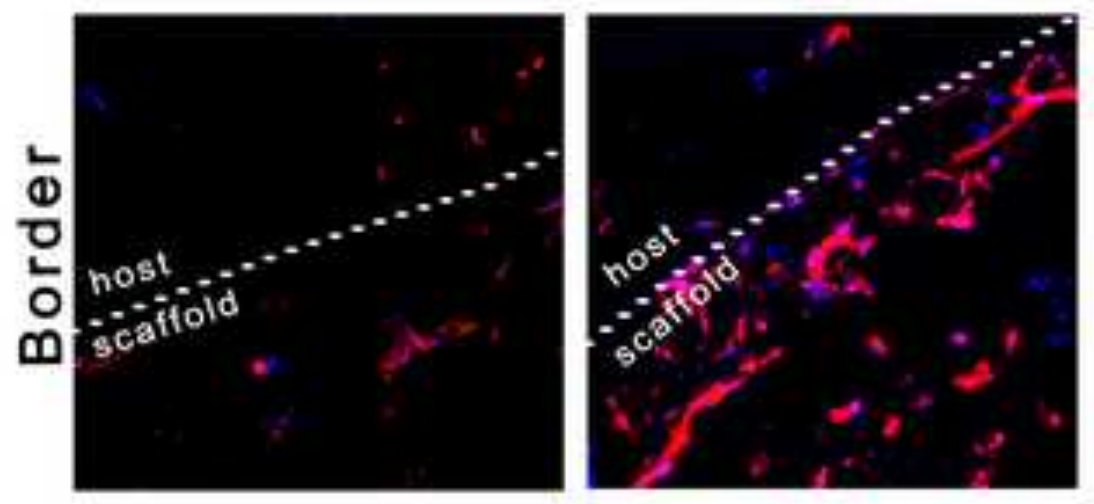

C

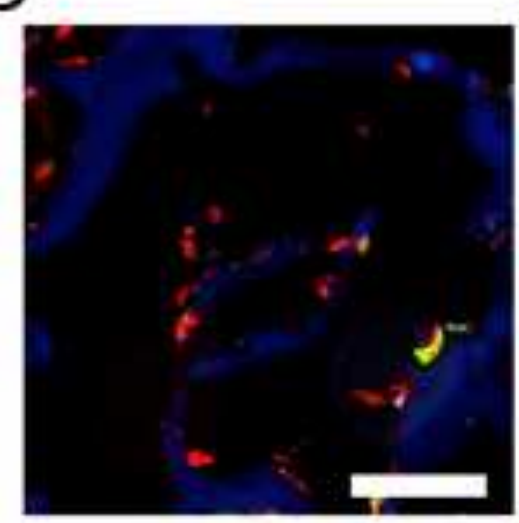

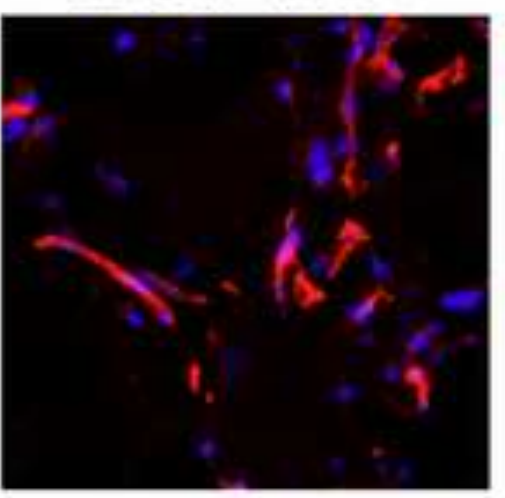

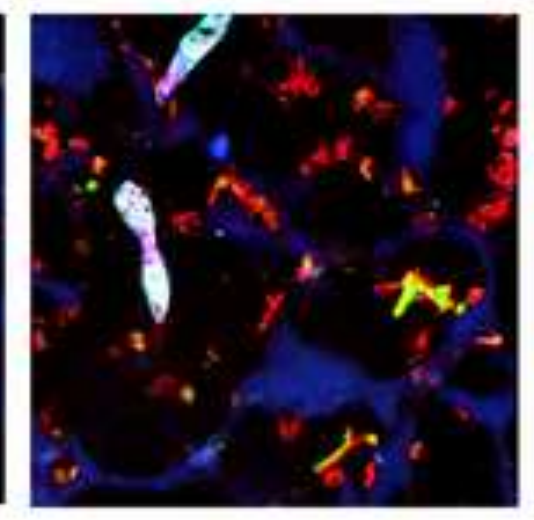


A

Naive ASC

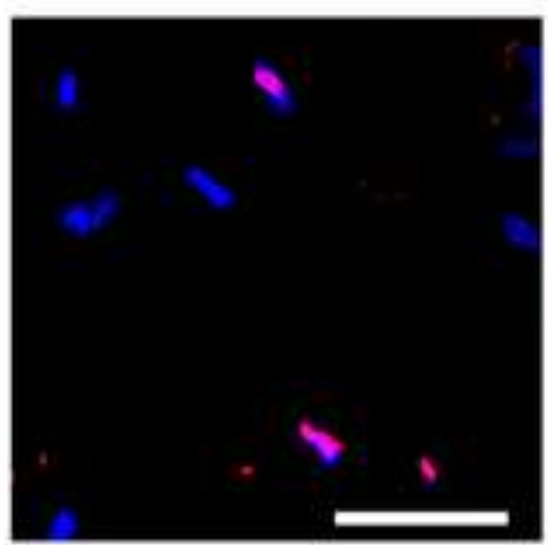

C

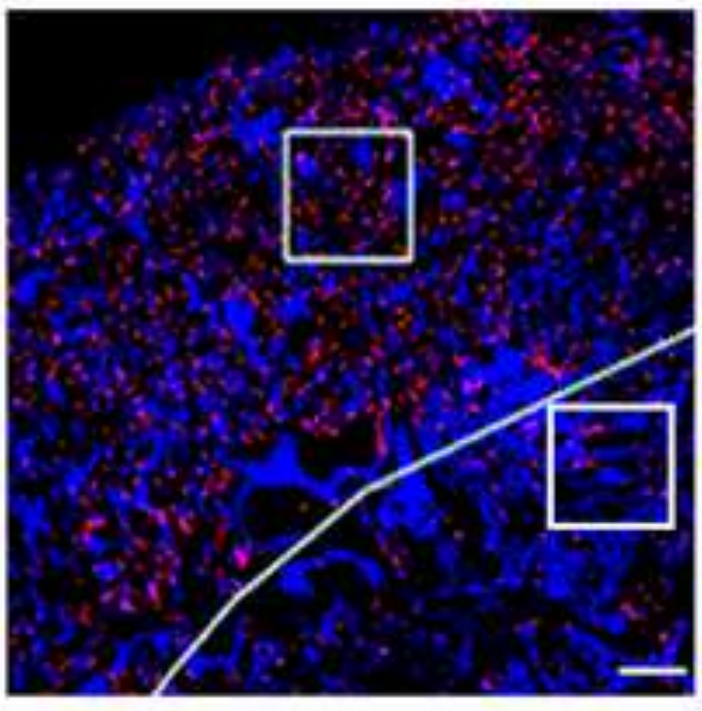

E

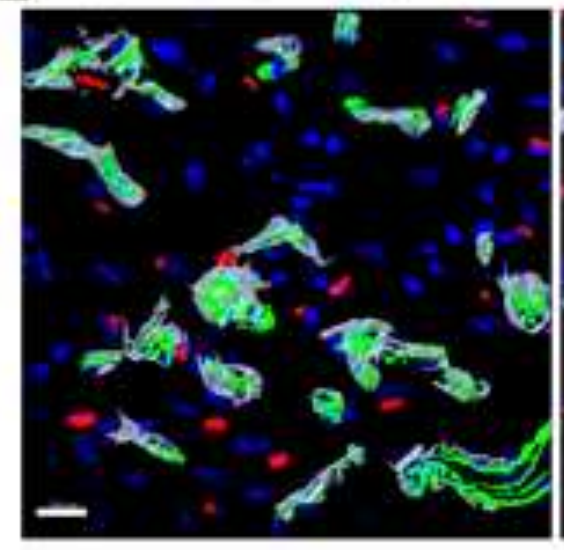

VEGF-ASC

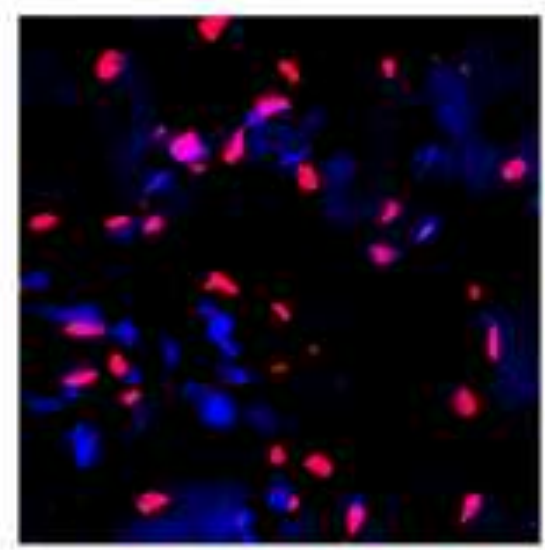

D
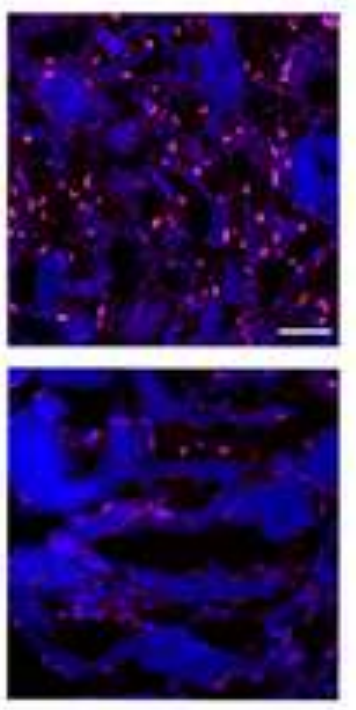

VEGF-ASC

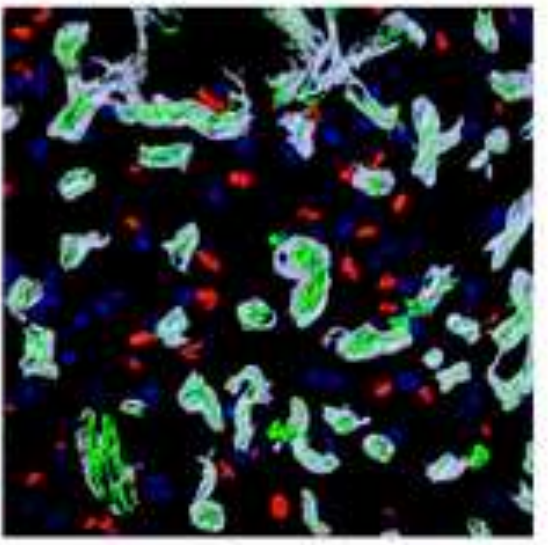

$\mathrm{F}$
Patch
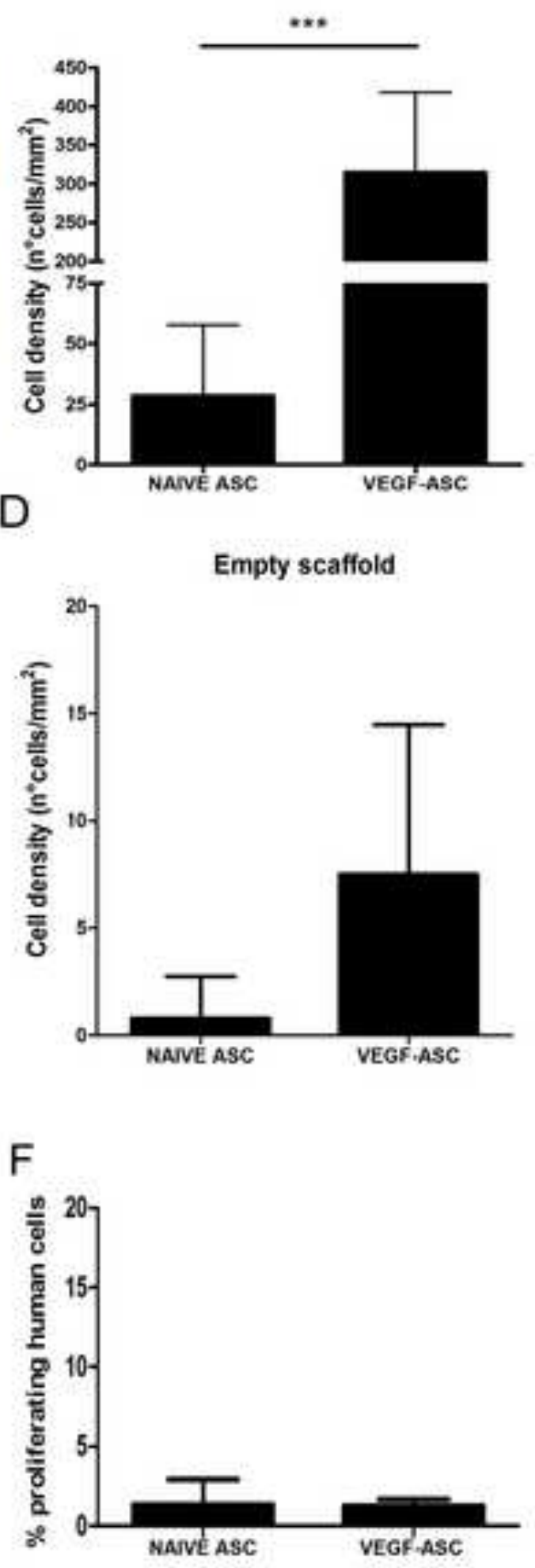